\title{
Tinea Capitis Inflamatoria por Trichophyton tonsurans var. sulfureum
}

\author{
(Inflammatory tinea capitis by Trichophyton tonsurans var. sulfureum)
}

Aida A. van Gelderen*.

Cátedra Micología, Instituto de Microbiología «Luis C. Verna», Facultad de Bioquímica, Química y Farmacia, Universidad Nacional de Tucumán. Ayacucho 491, (4000) San Miguel de Tucumán, R. Argentina.

Tel: $54-381-4247752$

*Autor para correspondencia: micologia@ fbqf.unt.edu.ar

Recibido:19-11-12

Aprobado:03-12-12

Palabras clave: Kerion Celsi, Tinea capitis, Trichophyton tonsurans.

Key words: Kerion Celsi, Tinea capitis, Trichophyton tonsurans.

\section{RESUMEN}

Los dermatofitos constituyen un grupo de hongos queratinofílicos y queratinolíticos que pueden producir lesiones en piel y sus anexos en animales y el hombre. Las lesiones pueden variar de intensidad desde leves a severas, algunas de las cuales son altamente inflamatorias. Trichophyton tonsurans es un dermatofito antropofílico agente de tiñas no inflamatorias, de piel y raramente de uñas. Es altamente contagioso, que se adquiere por contacto interhumano y de escasa presentación en nuestro medio. Raramente y asociado a estados de inmunocompromiso ocasiona tiña inflamatoria de cuero cabelludo (Kerion Celsi o Querión de Celso). Kerion Celsi es una lesión altamente inflamatoria y supurativa, generalmente causada por dermatofitos zoofílicos que se transmiten de animales al hombre, la cual representa una respuesta inmune exagerada del huésped a la presencia del hongo.

Se documenta un caso de Kerion Celsi a $T$. tonsurans presentado por un niño de 4 años de edad, sin inmunocompromiso, residente en el interior de la provincia. Se trata del primer caso local de tinea capitis altamente inflamatoria a $T$. tonsurans. El propósito es demostrar que el hongo, a pesar de ser antropofilico, puede ocasionar esta forma clínica en paciente sin inmunocompromiso. Su existencia en el medio requiere de diagnóstico rápido, de extremar medidas higiénicas y posterior control para evitar su propagación.

\section{ABSTRACT}

The dermatophytes are keratinophilic and keratinolytic fungi that cause skin and its annexes lesions in animals and man. T. tonsurans is an anthropophilic fungi, highly contagious, of rare presentation in our environment. Infection is acquired by interpersonal. Noninflammatory ringworm occurs in children and adults. Very rarely it causes highly inflammatory and suppurative ringworm of the scalp (Celsi kerion) associated with immunocompromised states. The kerion is caused by zoophilic dermatophytes. It represents an exaggerated host immune response to the presence of the fungus.

This paper presents a case of $T$. tonsurans Kerion in a 4-year-old non-immunocompromised patient, who lives in rural area in the province. This is the first local case of severe inflammatory tinea capitis to $T$. tonsurans. The purpose of the study is to demonstrate that $T$. tonsurans can cause Kerion not associated to immunocompromised. 
Furthermore, it shows its existence in the medium, which requires immediate diagnosis of the diseases and increase hygiene and disease control to prevent the spread of the fungus.

\section{INTRODUCCIÓN}

La tiña de la cabeza (tinea capitis) es una enfermedad que afecta especialmente a la población infantil. Su frecuencia y etiología varían en diferentes partes del mundo. La mayoría de los casos curan espontáneamente en la pubertad debido a cambios hormonales que modifican la secreción sebácea y el pH que actúan como fungistáticos. Los casos de adultos se asocian, generalmente a estado de inmunocompromiso (1-9).

Los dermatofitos son hongos queratinofílicos y queratinolíticos. Según su habitat pueden ser geofilicos, zoofílicos 0 antropofilicos. Algunos producen lesiones humanas en piel y anexos y en animales. Las lesiones varían desde leves a severas $y$ algunas son altamente inflamatorias $(1,2,6,7,9)$.

T. tonsurans es un dermatofito antropofilico, altamente contagioso, de escasa presentación en nuestro medio. Se adquiere por contacto interhumano y por contacto con materiales contaminados. Es agente de tinea capitis no inflamatoria, generalmente en niños de corta edad $\mathrm{y}$, con menor frecuencia, de tinea corporis y tinea unguiun. La lesión que produce en cuero cabelludo es lesión localizada que puede complicarse si se infecta secundariamente por bacterias y extenderse si existe alguna enfermedad de base debilitante. El hongo invade el estrato córneo y los orificios de los folículos pilosos entrando en este y creciendo a lo largo y debajo de la cutícula, produciendo una abundante esporulación en el interior del pelo que hace que éste se rompa $(1,2,6,7,9)$. La lesión, si bien tiende a desaparecer en la pubertad, puede extenderse en la vida adulta, a diferencia de otras tiñas dermatofíticas $(2,9,10)$.

T. tonsurans tiene distribución mundial. Se lo describió como del Viejo Mundo donde prácticamente esta erradicado. Es endémico en México, en otros países latinoamericanos y en las islas del Pacífico Sur. Tiene prevalencia muy elevada en EEUU donde se lo ha considerado un problema de salud pública y es emergente en otras regiones $(4,7-10)$.

Kerion Celsi o Querión de Celso es una lesión fúngica del cuero cabelludo altamente inflamatoria y supurativa. Es originada por dermatofitos poco adaptados al parasitismo humano, generalmente zoofílicos que se transmiten de animales al hombre y raramente por especies geofísicas. En consecuencia despiertan en el huésped una respuesta inflamatoria más violenta que las especies antropofílicas, respuesta de hipersensibilidad de tipo IV frente a los antígenos fúngicos (11). Son lesiones bien circunscritas, con pelos rotos o que se eliminan completamente, generalmente húmedas, que drena pus por múltiples folículos pilosos, dolorosas, con prurito escaso y adenomegalias, pudiendo afectar el estado general y, a veces, ocasionar alopecia residual. $(1-3,8,9,12,13)$. El Kerion también se ha visto asociado con eritema nodoso (14).

En el mundo hay pocos casos severos de Kerion a $T$. tonsurans, lo cual ocurre asociado a estado de inmunodepresión (13, 15-17).

Se comunica un caso de Kerion Celsi a $T$. tonsurans en un niño con residencia en el interior de la provincia de Tucumán (Argentina), quien presentó una lesión altamente inflamatoria con características particulares, no comunes a los Kerion de otros dermatofitos. El objetivo es poner en evidencia que este hongo antropofilico también puede ocasionar tiña altamente inflamatoria en pacientes inmunocompetentes, resaltar la importancia del diagnóstico precoz y contribuir al conocimiento de la enfermedad en el medio.

\section{CASO CLÍNICO}

Paciente de sexo masculino de 4 años de edad, procedente del interior de la provincia, de buen estado general y sin antecedentes de inmunocompromiso. Concurrió al laboratorio de Diagnóstico de la Cátedra Micología de la Facultad de Bioquímica, Química y Farmacia de la Universidad Nacional de Tucumán, en el que se registra con el $\mathrm{N}^{0} 7253$. Presentaba una lesión única en la cabeza comprometiendo el cuero cabelludo y los pelos, que según el relato de su 
madre, tenía 4 días de evolución. Había sido evaluado previamente y le prescribieron antibacterianos sistémicos sin ninguna mejoría. Comenzó como placa pseudoalopécica muy delimitada, alcanzando los $4 \times 4 \mathrm{~cm}$, irregularmente redondeada, con eritema y descamación. Seis días más tarde mostraba una lesión más extendida y recubierta de costras hemáticas.La zona lesionada mostraba marcada inflamación, numerosas pústulas y secreción purulenta a nivel de los folículos pilosos.Progresó de forma aguda y dolorosa (Figuras 1 ). A los 14

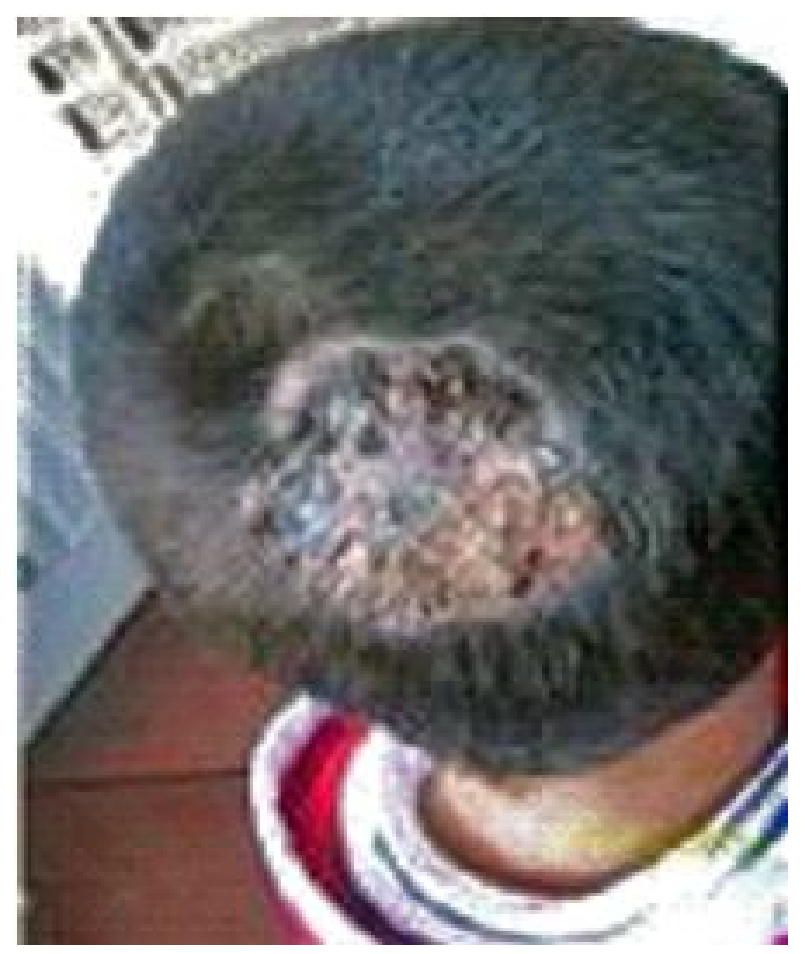

Figura 1. Kerion a T. tonsurans de 10 días de evolución, mostrando numerosas pústulas y costras hemáticas.

\section{ESTUDIO MICOLÓGICO}

De la zona periférica de la lesión se recogieron pelos del cuero cabelludo en forma aséptica y fueron colocados entre portaobjetos estériles. Con material purulento se practicaron cultivos.

Para el examen de los pelos se realizaron preparaciones con solución alcalina ( $\mathrm{KOH}$ al $20 \%$ ), entre porta y cubreobjetos con ligero calentamiento, días de evolución y bajo tratamiento oral con griseofulvina (10 mg/kg/día) aún mostraba edema franco y secreción, con escasos pelos en su extensión.

A los 24 días de evolución el tratamiento oral fue suspendido por presentar modificación del nivel de enzimas hepáticas, continuando con tratamiento local exclusivamente. Curó a los 60 días, a partir de los cuales se empezó a hacer evidente la aparición de los primeros pelos.

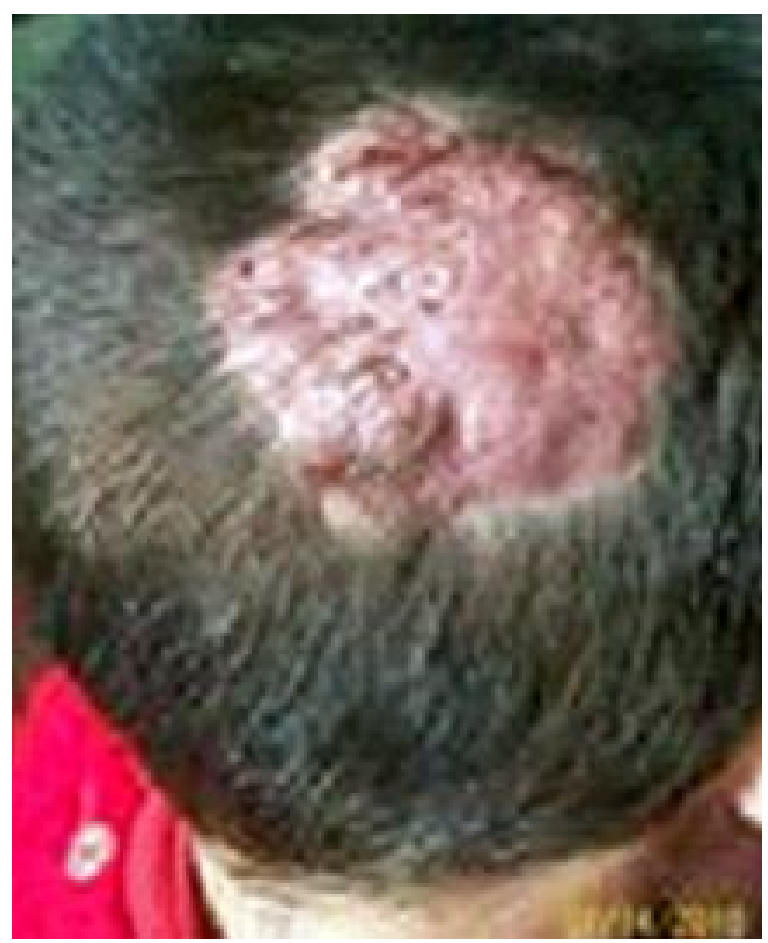

Figura 2. Lesión con 14 días de evolución y bajo tratamiento con griseofulvina. Marcado edema y lesiones supurantes con ausencia de pelos en algunas zonas.

para aclarar la queratina del material. El examen de pelos mostró numerosos esporas megasporados (de alrededor de $8 \mu$ de diámetro) en crecimiento endotrix (invasión del pelo con producción de artroconidias en su interior) (Figura 3).

Se practicaron cultivos de los materiales clínicos en medio Sabouraud- glucosado-agar con extracto de levaduras y cloranfenicol (SAB) y en medio de aislamiento Dermatophytes Test Media 
(DTM) que contiene rojo fenol como indicador de pH para poner en evidencia productos metabólicos del hongo que alcaliniza al medio dando un color rojo violeta, al igual que otros dermatofitos. La incubación fue realizada a $28^{\circ} \mathrm{C}$ durante 15 días. En
SAB desarrollaron colonias de aspecto finamente pulverulentoas, de color amarillo azufre y reverso pardo rojizo. Después de 7 días de incubación pudo observarse el cambio de color del medio DTM producido por su alcalinización (Figura 4).

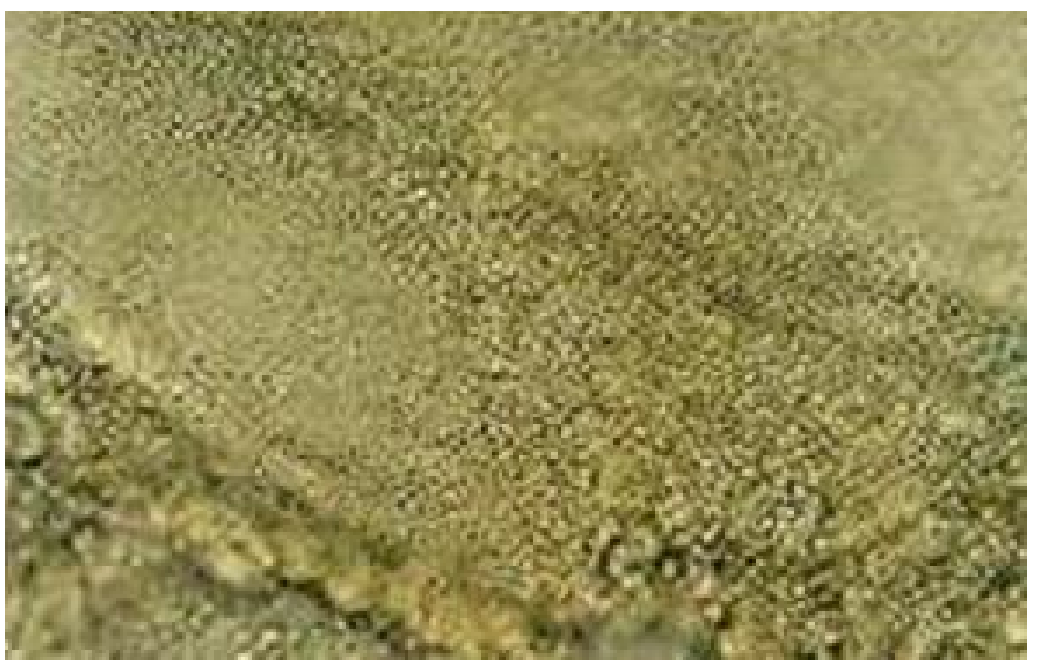

Figura 3. Pelos parasitados por $T$. tonsurans mostrando abundantes esporas megasporados

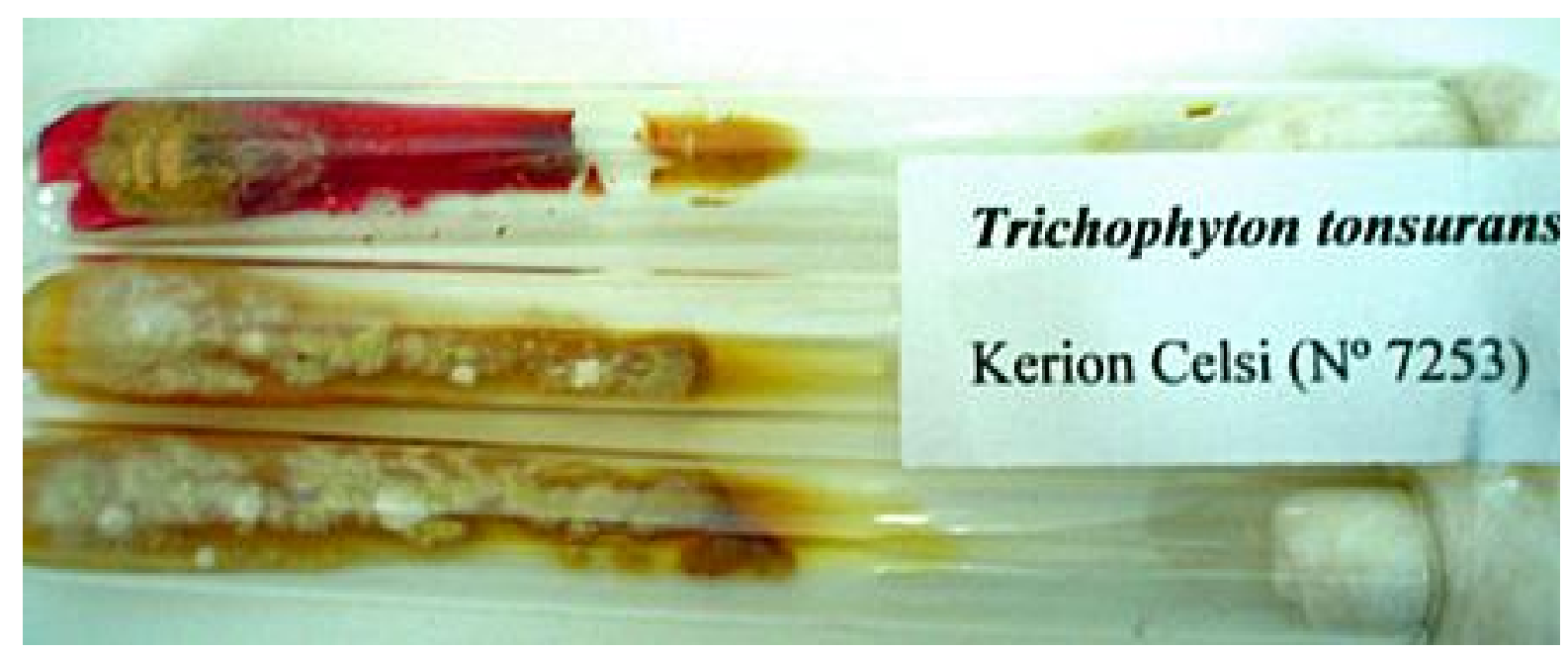

Figura 4. Cultivos de T. tonsurans en medios DTM (arriba), mostrando el cambio del color del medio a rojo violáceo y en $S A B$ (abajo)

La observación microscópica de los cultivos mostró hifas septadas, hialinas, que no se tiñeron uniformemente con el azul de lactofenol usado para las preparaciones microscópicas. En cultivos jóvenes (de 7-10 días) se observaron numerosas microconidias ovoides, alargadas, piriformes, de clava 0 de lágrimas, 0 las típicas hinchadas en forma de balón $(4-8 \times 1-3 \mu)$ sésiles 0 sobre cortos conidióforos a lo largo de las hifas o en racimos. Las microconidias fueron escasas, de pared fina, de formas y tamaño variables $(2-5 \times 3-7 \mu)$, predominando las de forma de cigarro sobre las cilíndricas, generalmente con 2 a 3 células. En cultivos más viejos se observaron masas de clamidoconidios.

La identificación del aislamiento fue realizada por estudio de sus caracteres macro y micromorfológicos y por pruebas fisiológicas que valoran su crecimiento y sus requerimientos nutricionales: producción de ureasa, perforación del 
pelo in vitro y determinación del requerimiento de tiamina para su crecimiento, Para determinar la producción de ureasa se usó el medio de Christensen, para la perforación del pelo in Vitro se siguió la técnica de Vanbreuseghem y para el estudio de sus requerimientos nutricionales los agares Trichophyton (Difco) siguiendo a Rebell y Taplin, 1970, Rippon, 1988 y De Hoog et al., 2000 (1, 3, 5). El aislamiento de $T$. tonsurans produjo ureasa, no perforó pelos in vitro y tuvo buen crecimiento en medio con tiamina. Fue identificado como $T$. tonsurans var. sulfureum.

\section{DISCUSIÓN}

T. tonsurans es de baja presentación en nuestro medio, casi siempre como agente de tinea capitis. Un análisis de 366 casos de tinea capitis diagnosticadas en el Servicio de Diagnóstico de la Cátedra de Micología, revela solo 12 casos (3,1\%) producidos por $T$. tonsurans, aislados y en brotes entre los años 1980-1984 (17). Este porcentaje es semejante al de 3,2 \% obtenido por García Diaz y colab. y menor al de 8,34 \% informado por Davel y colab., ambos correspondientes a Buenos Aires (18, 19). En Tucumán, la mayoría de los Kerion fueron causados por dermatofitos zoofilicos ( $M$. canis, $T$. mentagrophytes var. mentagrophytes y $M$. gypseum) representando el 4,37 \% de las tiñas de la cabeza (17).

Se conoce que $T$. tonsurans es causante de brotes familiares y nosocomiales, pudiendo existir portadores asintomáticos del hongo $(6,7,12,13$, $18,21,22)$, lo cual señala la importancia del control de la enfermedad para limitar su propagación por contagio interhumano y por materiales contaminados.

Por su condición de hongo antropofilico, sería de esperar que $T$. tonsurans ocasione tiña noinflamatoria en el hombre inmunocompetente, el cual tendría que estar habituado a su contacto. Sin embargo, asociado a estados de inmunodepresión, es agente de Kerion (2, 9, 13, 18, 21). Este caso severo de Kerion a $T$. tonsurans variedad sulfureum representa el primero altamente inflamatorio observado en la región y uno de los pocos del mundo $(18,23)$, a pesar que, en los casos de tinea capitis a $T$. tonsurans diagnosticados en Tucumán, esta variedad es la predominante (17). Las costras hemáticas y la gran inflamación le atribuyeron características clínicas particulares, semejantes a algunos de los casos descritos por Rodríguez y colab. (13). A la variedad sulfureum se la relaciona con la producción, en el cuero cabelludo, de múltiples lesiones tipo Kerion y eritema nodoso $(4,23)$. La ausencia de inmunocompromiso del paciente y su buen estado general, hacen suponer que la lesión podría deberse, además de la sensibilidad a los productos metabólicos del hongo, a factores de la cepa, como mayor agresividad. Se hace imprescindible la correcta identificación de todo aislamiento de tinea capitis, los altamente inflamatorios requieren de un diagnóstico precoz que contribuya a restringir la lesión y la aparición de alopecia cicatrizal permanente por destrucción de los bulbos pilosos de la zona lesionada.

La documentación de este caso fue considerada de importancia por lo infrecuente del agente etiológico y, por las particularidades clínicas del caso, para orientar al diagnóstico micológico. También es necesario su reconocimiento en el ambiente médico, por la particular presentación de la enfermedad inflamatoria, para evitar un diagnóstico erróneo al ser interpretado como afección de origen bacteriano.

\section{AGRADECIMIENTO}

Trabajo subsidiado por el Consejo de Investigaciones de la Universidad Nacional de Tucumán, Proyecto 26 D/454 (Directora: Dra. Aida van Gelderen).

\section{REFERENCIAS}

1. Rebell,G.; Taplin,D. (1970). The Dermatophytes. 2nd. revised edition. University of Miami Press, Coral Gables, Florida. USA.

2. Kwon-Chung,K.J.; Bennett,E. (1992) .Medical Mycology. Philadelphia: Lea \& Febigen. 
3. Rippon,J.W. (1998). Medical Mycology (3a ed). Philadelphia: WB Saunders Company.

4. Arenas,R.; Torres,E.; Amaya,M.; Rivera,E.R.; Espinal,A.; Polanco,M.; Fernández,R.; Isa-Isa,R. (2010). Tinea capitis. Emergencia de Microsporum audouinii y Trichophyton tonsurans en la República Dominicana. Actas Dermato-Sifiliográficas 101(4): 330-335.

5. De Hogg,G.S.; Guarro,J.; Gené,J.; Figueras,M.J. (2000). Atlas of Clinical Fung ( $\left(2^{\mathrm{a}} \mathrm{ed}\right)$.

6. Kane,E.J. (1997). Laboratory handbook of dermatophytes. Star Publishing Company.

7. Moraes,M.S.; Godoy-Martinez,P; Alchomme,M.M.; Boatto,H.F.; Fischman O. (2006). Incidence of Tinea capitis in São Paulo, Brazil. Mycopathologia 162(2):91-95.

8. Weitzman,I, Summerbell,R.C. (1995). The dermatophytes. Clin Microbiol Rev 8:240-259.

9. Georg, LK. (1952). Trichophyton tonsurans Ringworm: A New Public Health Problem Public. Health Reports 67 (1): 53-56.

10. Leeming,J.G.; Elliott,T.S. (1995). The emergence of Trichophyton tonsurans tinea capitis in Birmingham, U.K. $\mathrm{Br} J$ Dermatol. 133(6):929-931.

11. Lynch,P.; Finquelievich,J.; Etchepare,P.; Lamy,P.;Ré,R.; Litwak G.; Diaz,V.; Desseta,M.; Medina,E.;Casabé,S. (2005). Tinea capitis: estudio epidemiológico en el Hospital Municipal Materno Infantil de San Isidro «Dr. C. Gianantonio» (periodo Abril 2000 a Marzo 2002) Dermatol.Pediatr.Lat. 3(1): 39-43.

12. Bode,U.; Dorm,M.; Ring,J. (1980). Kerion celsi. Clinical aspects and pathogenesis of deep trychophytosis of the scalp. Fortschritte der Medizin 98(35):1348-1350.

13. Rodríguez,M.; Padilla,M.DelC.; Martínez,J.Á. (2006). Tiña inflamatoria de la cabeza por
Trichophyton tonsurans. Comunicación de 5 casos dentro de un mismo núcleo familiar. Rev. Cent. Dermatol.Pascua 15(1): 26-31.

14. Calista,D.; Schianchi,S.; Morri,M. (2001). Erythema nodosum induced by Kerion Celsi of the scalp. Pediatr Dermatol. 18(2):114-116.

15. Arreaza,E.; Arreaza,F.; Mizrachi,R.(1990). Tiña inflamatoria en el cuero cabelludo a Trichophyton tonsurans / Inflammatory tinea in the scalp of the left parietal region a Trichophyton tonsurans. Progresos en Ciencias Médicas 4(3):32-33.

16. O‘Donnell,B.; Powell, F.; Hone,R.; O'Loughling,S. (1990). Kerion-clinical spectrum in nine cases. Irish Journal of Medical Sciences 159 (1): 14-18.

17. Moraes,M.S.; Godoy-Martinez,P.; Alchomme,M.M.; Boatto,H.F.; Fischman O. (2006). Incidence of Tinea capitis in São Paulo, Brazil. Mycopathologia 162(2):91-95.

18. van Gelderen,A.; Borges de Kestelman,I. (2004). Epidemiological analysis of 385 human cases of tinea capitis. Casuistry by the Mycology Department. Abstract publicado en BIOCELL, 28(1): 80.

19. García-Diaz,R.; González, M.T.; Santos,P.; Pierini, A.M. (1995). Superficial Mycoses in children: a 3-year survey in Buenos Aires. En: Pierini AM, García-Diaz,R.; Bustamante, R.E. editores. Pediatric Dermatology, Buenos Aires, Elsevier, p75-78.

20. Davel,G.; Perrotta,D.; Canteros,C.; Córdoba,L.; Rodero,L.; Brudny,M. et al. (1999). Estudio multicéntrico de micosis superficiales en Argentina. Rev. Argent. Microbiol.. 31:173-181.

21. White,J.M.; Higgins,E.M.; Fuller,L.C. (2007). Screening for asymptomatic carriage of Trichophyton tonsurans in household contacts of patients with tinea capitis: results of 209 patients 
from South London. J.Eur.Acad.Dermatol. Venereol. 23. Kligman,A.M.; Constant, E.R. (1951). Family 21(8):1061-1064. epidemic of tinea capitis due to Trichophyton tonsurans (variety sulfureum). Arch.Dermat.\& Syph.

22. Lewis,S.M. \& Lewis,B.C. (1997). Nosocomial 63: 493-499).

transmission of Trichophyton tonsurans tinea corporis in a rehabilitation hospital. Infect Contr.Hosp. Epid.18:322-325. 